\title{
Pectus excavatum como debut de Síndrome de Marfan. Reporte de caso
}

Pectus excavatum as debut of Marfan Syndrome: case report -

Puglla Sánchez Luis René ${ }^{1}$, Sinche Cueva Cristian Andrés², Pérez López Jhonattan Sebastian ${ }^{3}$, Quilismal Guanochanga Nasthia Coraly ${ }^{4}$

VOLUMEN 38 | N³ | DICIEMBRE 2020

FECHA DE RECEPCIÓN: 14/06/2020 FECHA APROBACIÓN: 03/03/2021 FECHA DE PUBLICACIÓN: 06/04/2021

1. Médico en libre ejercicio. Quito - Ecuador

2. Médico en libre ejercicio. Loja - Ecuador

3. Médico en libre ejercicio. Ambato - Ecuador

4. Postgradista de la Universidad de la República - Hospital de Clínicas Dr. Manuela Quintela. Especialidad Endocrinología. Montevideo - Uruguay

\begin{tabular}{c|c} 
Caso & $\begin{array}{c}\text { Clinical } \\
\text { Case }\end{array}$ \\
\hline
\end{tabular}

DOI: https://doi.org/10.18537/RFCM.38.03.07

Correspondencia:

luispu_1994@hotmail.com

Dirección:

Av. Gaspar de Villarroel y Av. 6 de Diciembre

Código Postal:

170501

Teléfono:

0994566734

Quito - Ecuador

\section{RESUMEN}

Introducción: el Síndrome de Marfan (SM) es un trastorno autosómico dominante caracterizado por alteraciones del tejido conectivo; en el que la talla alta, las extremidades y dedos alargados se asocian con anormalidades en los sistemas cardiovascular, esquelético y ocular. Su diagnóstico se basa en los criterios de Ghent, que reúne hallazgos clínicos y factores hereditarios. Su principal complicación catastrófica es la dilatación y disección de aorta. Mediante el análisis del presente caso clínico se espera recabar las principales características relacionadas a esta importante patología que aporte argumentos claves a la literatura médica para un diagnóstico precoz y seguimiento oportuno que disminuya la morbimortalidad de esta enfermedad.

Caso clínico: presentamos un paciente masculino en seguimiento por parte de Medicina Interna, por su Pectus Excavatum (PE); además, presenta estrías cutáneas, signos de la muñeca y pulgar, escoliosis dorsal, proporción reducida del segmento superior/inferior, índice brazada/estatura aumentada y prolapso de válvula mitral, sumando un puntaje total de 8 en la escala de Ghent se confirma diagnóstico de SM.

Conclusiones: el SM presenta una clínica heterogénea, cuyo diagnóstico se basa en los criterios de Ghent. Existe un alto riesgo de complicaciones aórticas, con alta tasa de mortalidad por lo que el diagnóstico temprano es fundamental.

Palabras clave: tórax en embudo, síndrome de Marfan, válvula mitral. 


\section{ABSTRACT}

Introduction: Marfan syndrome (MS) is an autosomal dominant disorder characterized by connective tissue disorders; in which tall stature, elongated limbs and fingers are associated with abnormalities in the cardiovascular, skeletal, and ocular systems. Its diagnosis is based on the Ghent criteria, which combines clinical findings and hereditary factors. Its main catastrophic complication is dilation and dissection of the aorta. Analyzing a clinical case, it is hoped to collect the main characteristics related to this important pathology that provide key arguments to the medical literature for an early diagnosis and timely follow-up that reduce the morbidity and mortality of this disease.

Clinical case: a male patient who was monitoring by Internal Medicine was presented in this medical case, due to his pectus excavatum (PE), he also presents skin striae, wrist and thumb signs, dorsal scoliosis, reduced proportion of the upper / lower segment, stroke index / increased stature and mitral valve prolapse, adding a total score of 8 on the Ghent scale confirms the diagnosis of MS.

Conclusions: MS presents a heterogeneous clinical picture, whose diagnosis is based on the Ghent criteria. There is a high risk of aortic complications, with a high mortality rate, so early diagnosis is essential.

Key words: funnel chest, Marfan syndrome, mitral valve.

\section{INTRODUCCIÓN}

El SM es un trastorno hereditario del tejido conectivo con una incidencia estimada de uno por cada 5000 individuos. Esta patología fue descrita inicialmente por el parisino BernardJean Antoine Marfan en 1896 y se trasmite como un carácter autosómico dominante ${ }^{1}$.
El 49\% de los pacientes con diagnóstico de SM presentan un antecedente familiar positivo para esta patología, mientras que en un 30\% de ellos ocurre de manera espontánea, teniendo que considerar la posibilidad de una mutación de novo. El defecto es causado por la mutación del gen FBN1, que codifica para la proteína fibrilina-12. Esta glicoproteína es el principal componente de las fibras elásticas del tejido conectivo con una amplia distribución en la matriz extracelular de la piel, pulmones, vasos sanguíneos, riñones, cartílago, tendón, músculo y córnea. Otra mutación que puede estar presente es la del gen TGFBR2 que codifica para el receptor II del factor de crecimiento transformante beta y que ha sido asociado con el SM tipo 2; sin embargo, el hallazgo de esta alteración ha sido raro ${ }^{1,3}$.

Las manifestaciones clínicas típicas se localizan a nivel ocular, con ectopia lentis, miopía o desprendimiento de retina; a nivel esquelético, con deformidades torácicas, aracnodactilia, escoliosis o hiperlaxitud articular; manifestaciones cardiovasculares como la insuficiencia aórtica y mitral, aneurismas y disección aórtica, así como endocarditis bacteriana ${ }^{4}$. Entre las deformidades torácicas el PE constituye una de las principales características fenotípicas asociadas $^{5}$. 


\section{Tabla N¹}

Criterios Ghent revisados para diagnóstico de SM

\begin{tabular}{|c|c|}
\hline En ausencia de historia familiar de SM & En presencia de historia familiar (HF) de SM \\
\hline $\mathrm{Ao}(\mathrm{Z} \geq 2)+\mathrm{EL}=\mathrm{SM}$ & \multirow{3}{*}{$\begin{array}{l}E L+H F=S M \\
P u n t a j e \text { sistémico }(Z \geq 7 \text { puntos })+H F=S M \\
\text { Ao }(Z \geq 2 \text { por encima de los } 20 \text { años, } Z \geq 3 \text { si es } \\
\text { menor de } 20 \text { años })+H F=S M\end{array}$} \\
\hline $\mathrm{Ao}(\mathrm{Z} \geq 2)+\mathrm{FBN1}=\mathrm{SM}$ & \\
\hline $\begin{array}{l}\mathrm{Ao}(\mathrm{Z} \geq 2)+\text { puntaje sistémico }(\geq 7 \text { puntos })=\mathrm{SM} \\
\text { EL y FBN1 con aneurisma aórtico conocido = } \\
\text { SM }\end{array}$ & \\
\hline $\begin{array}{l}\text { Ao: puntaje Z del diámetro aórtico en senos de } \\
\text { FBN1: mutación en el gen de la fibrilina- } 1 \text {; Z: Z- }\end{array}$ & $\begin{array}{l}\text { alsalva o disección aórtica; EL: ectopia lentis; } \\
\text { ore: SM: síndrome de Marfan. }\end{array}$ \\
\hline
\end{tabular}

Fuente: Araújo, Marques, Freitas et al. ${ }^{7}$

El PE es una de las deformidades de la pared torácica más comunes, constituyendo el $88 \%$ del total de las mismas. Es importante recordar que el PE se encuentra incluido como un criterio mayor para el diagnóstico de $\mathrm{SM}^{6}$. El cual se basa en una serie de criterios clínicos y genéticos denominados nosología de Ghent (Tabla $N^{\circ} 1$ ). El hallazgo de las distintas manifestaciones clínicas del SM, actualmente determinan un puntaje de hallazgos sistémicos (Tabla N²) que se utiliza cuando el paciente presenta aortopatía, y la ectopia lentis está ausente ${ }^{7-9}$.
La identificación temprana y el manejo adecuado mejoran el pronóstico y la expectativa de vida de los pacientes con SM, quienes están propensos a complicaciones cardiovasculares. Por lo que es de gran importancia el conocimiento de las características de este síndrome al ser su diagnóstico netamente clínico. Su aporte educativo es valioso a la formación continua del médico, ya que el aprendizaje de la medicina se basa, generalmente, en la experiencia acumulada de casos clínicos lo cual motiva a su presentación.

\section{Tabla $\mathrm{N}^{\circ} 2$}

Puntaje de características sistémicas para SM presentes en el paciente

Signo de la muñeca y el pulgar: 3 puntos (signo de la muñeca o el pulgar: 1 punto)

Pectus carinatum: 2 puntos (PE:1 punto)

Deformidad en el retropié: 2 puntos (pie plano: 1 punto)

Neumotórax: 2 puntos

Ectasia dural: 2 puntos

Protrusión acetabular: 2 puntos

SS/SI reducida y proporción brazada/estatura incrementada (sin escoliosis grave): 1 punto

Escoliosis o cifosis toracolumbar: 1 punto

Extensión reducida del codo: 1 punto

Hallazgos faciales (3/5): 1 punto (dolicocefalia, enoftalmos, fisura palpebral baja, hipoplasia

malar, retrognatia)

Estrías cutáneas: 1 punto

Miopía mayor de 3 dioptrías: 1 punto

Prolapso mitral (todos los tipos): 1 punto

Total máximo 20 puntos; un puntaje de 7 o más indica afectación sistémica.

SS/SI: proporción segmento superior/inferior 
La dilatación aórtica se encuentra en un 80\% de pacientes con SM sobre todo si se asocian a $P E^{10}$. En el presente artículo se expone el caso de un paciente con PE con características fenotípicas de SM, con las que se obtiene el diagnóstico, sin la necesidad de recurrir a estudios genéticos debido al alto costo y la dificultad de realizarlos en nuestro país, considerando el porcentaje de pacientes con PE que concurren a consultas tanto de primer nivel como hospitalarias, es importante tener este diagnóstico presente con pacientes con dichas características.

\section{PRESENTACIÓN DEL CASO}

Paciente masculino de 26 años, nacido y residente en Loja, Ecuador, mestizo, educación superior completa. Antecedentes familiares: padre fallecido por enfermedad cardiovascular no especificada y nefropatía de Berger en ambos padres. Entre los antecedentes personales refiere que hace 4 años fue diagnosticado de subluxación bilateral del cristalino y aproximadamente hace 3 años mediante ecocardiografía se le diagnostica de prolapso de la valva mitral anterior sin presencia de insuficiencia valvular ni dilatación de grandes vasos.

El paciente acude a consulta externa del servicio de Medicina Interna del Hospital Isidro Ayora de la ciudad de Loja, Ecuador, para control por seguimiento de la deformidad torácica tipo PE (Imagen $\mathrm{N}^{\circ} 1$ ). El paciente refiere presentar deformidad a nivel de tórax desde el nacimiento, tornándose más evidente durante el crecimiento, sin evidencia concomitante de sintomatología alguna.

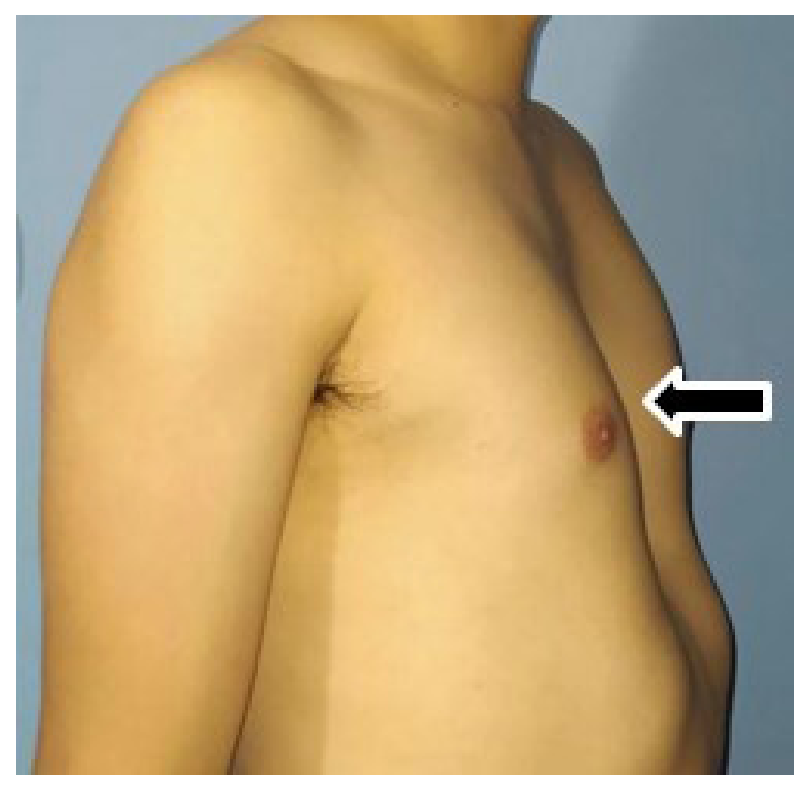

\section{Imagen $\mathrm{N}^{\circ} 1$}

Fotografía antero-lateral del tórax, se observa hundimiento central de la pared anterior del tórax (flecha)

Al realizar el examen físico se evidencia al paciente asintomático, orientado en tiempo, espacio y persona con TA: 95/65 mm/Hg, FC: 92 lat/min, FR: $18 \mathrm{rpm}$, temperatura: $36.5^{\circ} \mathrm{C}$, peso: $64 \mathrm{~kg}$, altura: $180 \mathrm{~cm}, \mathrm{IMC}$ : 19.75, de biotipo ectomorfo, la longitud de la brazada/envergadura es de $179 \mathrm{~cm}$. En la región torácica: a la auscultación cardíaca no se evidencia patología de importancia, presencia de deformidad anatómica tipo $\mathrm{PE}$, con expansibilidad torácica conservada; además, existe presencia de estrías atróficas (striae atrophicae) y escoliosis dorsal del tórax (Imagen $\mathrm{N}^{\circ} 2$ ). 


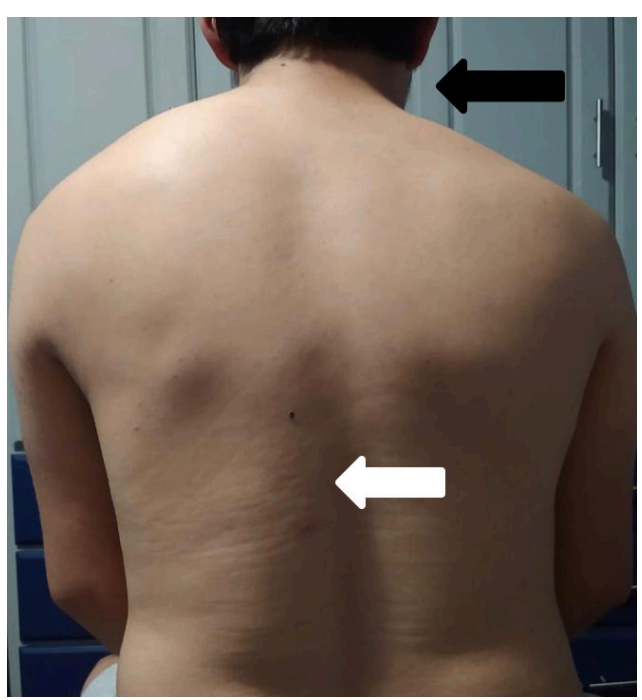

Imagen $\mathrm{N}^{\circ} 2$

Región dorsal, se evidencia estrías cutáneas (flecha blanca) y asimetría en hombros (flecha negra) debido a escoliosis dorsal.

En la evaluación de las extremidades se encuentra el signo de la muñeca (Imagen $\mathrm{N}^{\circ} 3$ ) y del pulgar positivo, presencia de dolicostenomelia con aracnodactilia (Imagen $\left.N^{\circ} 4\right)$; con una proporción segmento superior/ inferior reducida.

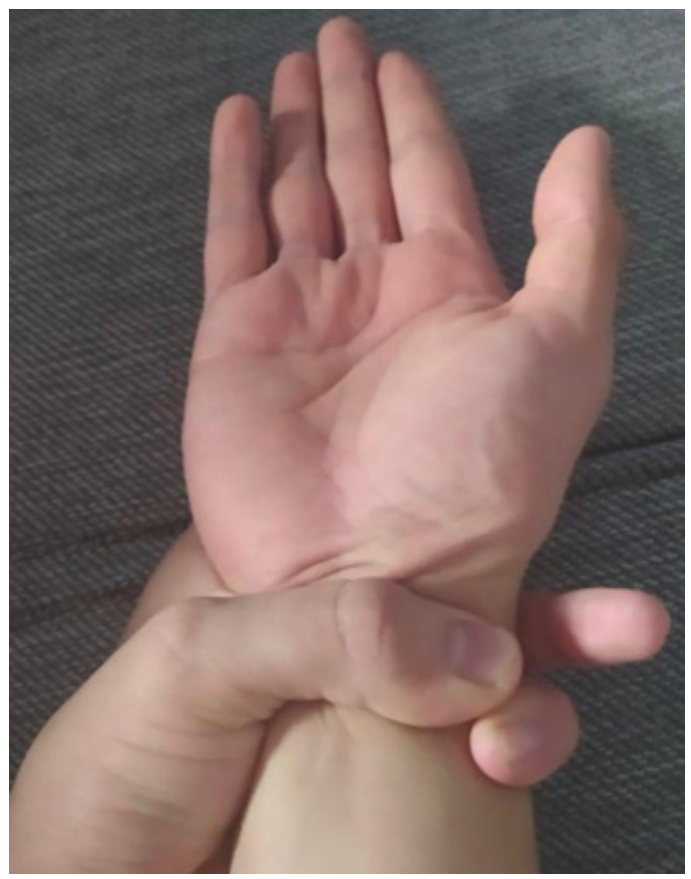

Imagen $\mathrm{N}^{\circ} 3$

Signo de la muñeca; la punta del pulgar cubre la uña entera del quinto dedo cuando envuelto alrededor de la muñeca contralateral.

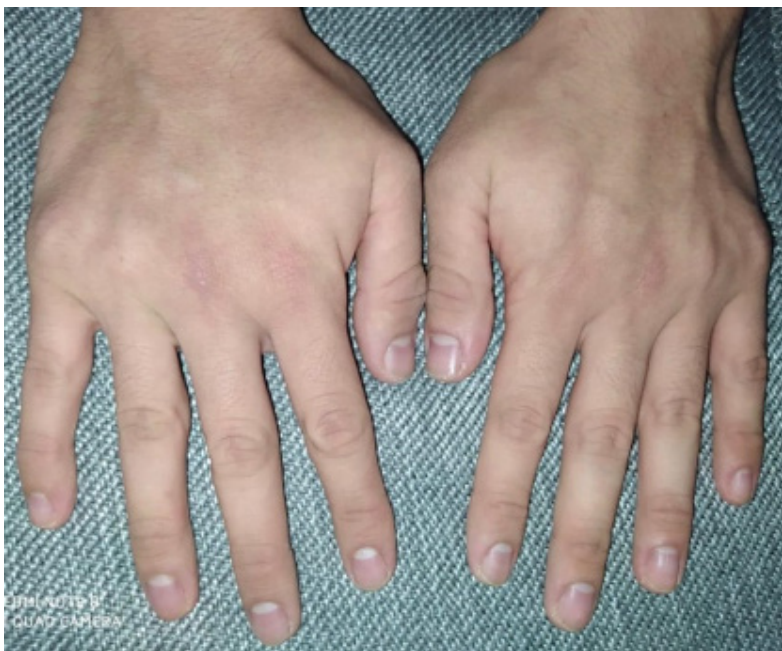

Imagen $\mathrm{N}^{\circ} 4$

Presencia de aracnodactilia (dedos de araña).

Los exámenes reportan: glicemia en ayunas $87 \mathrm{mg} / \mathrm{dL}$; colesterol total $175 \mathrm{mg} / \mathrm{dL}, \mathrm{C}-\mathrm{HDL}$ : 57 mg/dL, C-LDL: 101 mg/dL, triglicéridos: 105 $\mathrm{mg} / \mathrm{dL}$, BUN: $9.5 \mathrm{mg} / \mathrm{dL}$, plaquetas 232000 por $\mathrm{mcL}$, creatinina $0.7 \mathrm{mg} / \mathrm{dl}$, hemoglobina $16.9 \mathrm{~g} / \mathrm{dl}$, hemograma con 4900 leucocitos por $\mathrm{mcL}$, fórmula diferencial: granulocitos $56.9 \%$, monocitos $7.7 \%$, linfocitos $34.2 \%$. Se realiza ecocardiograma de control manteniendo como resultado el prolapso valvular mitral, sin insuficiencia valvular ni dilatación aórtica, no se encuentra hipertrofia de ventrículos.

Finalmente, con los antecedentes y hallazgos clínicos encontrados, pese a que no se realizó un estudio genético en búsqueda de la mutación específica por la limitación en la realización de estudios genéticos moleculares en el país; se llegó al diagnóstico de SM por presentar el antecedente familiar positivo y la presencia de características clínicas según los criterios de Ghent revisados, alcanzando un puntaje sistémico de ocho puntos. No se presentó dificultad para el diagnóstico en cuanto a barreras culturales, lingüísticas o de alguna índole. 
Tras el diagnóstico, se le recomendó al paciente realizar varias sesiones de terapia física y soporte ortopédico para la escoliosis. Además, el tratamiento oftalmológico para la subluxación del cristalino fue conservador mediante la prescripción de lentes. Se le propuso una intervención quirúrgica para la corrección estética del PE, la cual no se ha llevado a cabo hasta la actualidad por indecisión del paciente y falta de adherencia al tratamiento para sus comorbilidades. Se presentó al paciente el consentimiento informado, quien aceptó y permitió la publicación de sus datos clínicos e imágenes para la presentación de este caso. Actualmente el paciente acude a controles de manera periódica y se maneja un protocolo multidisciplinario con interconsultas y seguimiento por los servicios de genética clínica, traumatología, medicina interna y cardiología.

\section{DISCUSIÓN}

El SM es un trastorno sistémico del tejido conectivo. La enfermedad afecta principalmente los sistemas cardiovascular, ocular y esquelético. El diagnóstico se realiza basándose inicialmente en un minucioso examen clínico y una completa historia familiar, acompañada de ecocardiograma, evaluación oftalmológica y radiográfica ${ }^{4,6}$.

EI SM presenta una amplia variedad de fenotipos entre los desórdenes del tejido conectivo sistémico. Uno de los más característicos que ocurren en dos terceras partes de pacientes marfanoides, es la presencia de la deformidad en la pared torácica denominada PE ${ }^{11}$.

La progresión de la deformidad torácica en el PE ocurre durante la adolescencia, y por lo tanto en esta etapa es cuando los síntomas y los aspectos psicosociales pueden ser más evidentes $^{12}$. El Índice de Haller se utiliza para evaluar la gravedad de la deformidad del PE en la TAC (Imagen $\left.N^{\circ} 5\right)$. Tradicionalmente, un Índice de Haller de $>3.25$ se consideraba una indicación para la cirugía en pacientes con una deformidad del PE ${ }^{13,14}$.

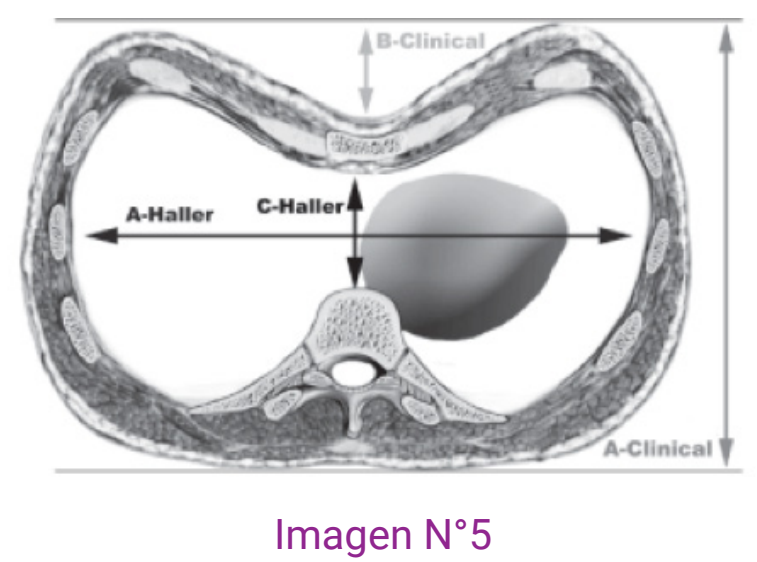

Esquema de una TAC de tórax, corte axial a nivel del tercio distal del esternón. Índice de Haller = A-Haller $/$ C-Haller (A-Haller = distancia latero-lateral máxima; $\mathrm{C}$-Haller $=$ distancia antero-posterior más corta). Fuente: Baldassari et al. ${ }^{14}$.

En el SM la principal complicación catastrófica es la dilatación y la disección de aorta que tiene mayor prevalencia en pacientes con $\mathrm{PE}^{14}$. Cuando ocurre la disección aórtica los pacientes presentan dolor abdominal agudo o dolor en el pecho y la espalda; no obstante, también puede presentarse atípicamente ${ }^{15}$. Otra característica fundamental entre pacientes con SM, constituyen la presencia de estrías cutáneas y la hiperlaxitud. La escoliosis constituye otro de los rasgos sistémicos presentes en el SM y su asociación en pacientes con PE es mayor ${ }^{16}$.

El resultado en cuanto a esperanza de vida mejora con el diagnóstico precoz, el manejo multidisciplinar comprendiendo revisiones oftalmológicas anuales, revisiones semestrales por el traumatólogo, en aquellos casos de escoliosis en adultos con deformidades progresivas, control por el cardiólogo y cirujano 
vascular mediante ecocardiogramas anuales y adjuntar el tratamiento médico para retrasar o prevenir la progresión de la dilatación aórtica y la cirugía electiva oportuna ${ }^{17,18}$.

\section{CONCLUSIONES}

El PE, una de las deformidades pectorales más comunes, se encuentra asociado a patologías del tejido conectivo como el SM. Se describe un caso de PE en control médico que presenta características identificables para el SM, basados en datos clínicos y antecedentes familiares, según criterios de Ghent. El SM tiene una presentación clínica muy heterogénea. La principal complicación catastrófica es la aortopatía, por afectar a la pared de la aorta. En este tipo de pacientes es imprescindible el diagnóstico precoz, un plan estratégico de seguimiento y prevención de las complicaciones.

\section{ASPECTOS BIOÉTICOS}

El presente caso clínico fue realizado bajo el consentimiento informado del paciente, con total confidencialidad de datos personales.

\section{INFORMACIÓN DE LOS AUTORES}

Puglla Sánchez Luis René. Médico. Libre ejercicio. Quito - Pichincha - Ecuador.

e-mail: luispu_1994@hotmail.com

ORCID:https://orcid.org/0000-0002-8433-8329

Sinche Cueva Cristian Andrés. Médico General. Libre ejercicio. Loja - Loja - Ecuador

e-mail: crisac27@gmail.com

ORCID:https://orcid.org/0000-0002-5842-1188

Pérez López Jhonattan Sebastián. Médico. Libre ejercicio. Ambato - Tungurahua - Ecuador.

e-mail: jhonattan.perez92@gmail.com

ORCID:https://orcid.org/0000-0002-4231-1953
Quilismal Guanochanga Nasthia Coraly. Médica. Postgradista de la Universidad de la República-Hospital de Clínicas Dr. Manuela Quintela. Especialidad Endocrinología. Montevideo - Montevideo - Uruguay.

e-mail: nastycoraly@hotmail.com

ORCID:https://orcid.org/0000-0002-1231-9962

\section{CONTRIBUCIÓN DE LOS AUTORES}

Todos los autores contribuyeron en la concepción y diseño del trabajo, así como la recolección de datos. Todos contribuyeron en la redacción, análisis crítico y revisión bibliográfica. Finalmente, todos los autores leyeron y aprobaron la versión final del manuscrito, respondiendo a todos los aspectos del mismo.

\section{CONFLICTO DE INTERESES}

Los autores declaran no tener conflicto de intereses alguno.

\section{FUENTES DE FINANCIAMIENTO}

Fondos propios

\section{REFERENCIAS BIBLIOGRÁFICAS}

1. Silbiger J, Parikh A. Pectus excavatum, echocardiographic, pathophysiologic, and surgical insights. Echocardiography [Internet]. Agosto 2016; [citado 11 julio 2020]; 33(8):1239-44. Disponible en: https:// pubmed.ncbi.nlm.nih.gov/27277386/

2. Pyeritz R. Marfan síndrome, improved clinical history results in expanded natural history. Genet Med [Internet]. Agosto 2019; [citado 2 agosto 2020]; 21(8):16831690. Disponible en: https://pubmed.ncbi. nlm.nih.gov/30573797/\#: :text=Life\%20 expectancy $\% 20$ for $\% 20$ a $\% 20$ person,features $\% 20$ of $\% 20$ the $\% 20$ skeletal\%20system 
3. Sandoval J, Saldarriaga W, Isaza C. Síndrome de Marfan, mutaciones nuevas y modificadoras del gen FBN1. latreia [Internet]. Junio 2014; [citado 23 Julio 2020]; 27(2):206-15. Disponible en: $\quad$ http://www.scielo.org.co/scielo. php? script=sci_arttext\&pid=S012107932014000200008\&lng=en

4. Wagner A, Zaradzki M, Arif R, Remes A, Müller O, Kallenbach K. Marfan síndrome, A therapeutic challenge for long-term care. Biochem Pharmacol [Internet]. Junio 2019; [citado 21 agosto 2020]; 164(2019):53-63. Disponible en: https://pubmed.ncbi.nlm. nih.gov/30926475/

5. Salik I, Rawla P. Marfan Syndrome. StatPearls [Internet]. Enero 2020; [citado 2 Agosto 2020]; p. 1-32. Disponible en: https://www.ncbi.nlm.nih.gov/books/ NBK537339/

6. Soto M, Cano R, Criales $C$, Avendaño L, Espínola N, García C. Pectus excavatum y carinatum en el síndrome de Marfan y síndromes similares, prevalencia e impacto clínico pulmonar y cardiovascular. Gac Med Mex [Internet]. 1 Enero 2018; [citado 6 Agosto 2020]; 154(2):67-78. Disponible en: https://pubmed.ncbi.nlm. nih.gov/30532108/

7. Araújo M, Marques C, Freitas S, SantaBárbara R, Alves J, Xavier C. Marfan Syndrome, new diagnostic criteria, same anesthesia care? Case report and review. Rev. Bras. Anestesiol [Internet]. Agosto 2016; [citado 6 julio 2020]; 66(4):408-413. Disponible en: https:// www.scielo.br/scielo.php?script $=$ sci $_{-}$ arttext\&pid=S0034-70942016000400408
8. Abdullah F, Harris J. Pectus Excavatum: More Than a Matter of Aesthetics. Ann. Pedriatric Surg [Internet]. 1 Noviembre 2016; [citado 17 Agosto 2020]; 45(11):4036. Disponible en: https://www.healio.com/ pediatrics/journals/pedann/2016-1145-11/\%7B532ace9d-c81a-4bab-b58fd5c2e53020ce\%7D/pectus-excavatummore-than-a-matter-of-aesthetics. pdf?fat=undefined

9. Behr C, Denning N, Kallis M, Maloney C, Soffer S, Romano-Adesman A, et al. The incidence of Marfan syndrome and cardiac anomalies in patients presenting with pectus deformities. J. Pediatr. Surg. [Internet] Septiembre 2019; [citado 19 Agosto 2020]; 54(9):1926-8. Disponible en: https://www.sciencedirect.com/science/ article/abs/pii/S0022346818308078

10. Fraser S, Child A, Hunt I. Pectus updates and special considerations in Marfan syndrome. Pediatr. Rep. [Internet] 21 Noviembre 2017; [citado 8 Agosto 2020]; 9:70-7. Disponible en: https://www.ncbi. nlm.nih.gov/pmc/articles/PMC5768089/

11. Abid I, Ewais M, Marranca J, Jaroszewski DE. Pectus excavatum, A review of diagnosis and current treatment options. J Am Osteopath Assoc [Internet]. 1 Febrero 2017; [citado 3 Agosto 2020]; 117(2):10613. Disponible en: https://pubmed.ncbi. nlm.nih.gov/28134952/

12. Tanaka Y, Matsumoto I, Saito D, Yoshida S, Takata M, Tamura M, et al. Surgical Treatment of Pectus Excavatum in Patients with Marfan Syndrome Associated with Vertebral or Cardiovascular Disease. Kyobu Geka [Internet]. Marzo 2020; [citado 21 Diciembre 2020]; 73(3):163-8. Disponible en: https://pubmed.ncbi.nlm. nih.gov/32393696/ 
13. Raffa GM, Kowalewski M, Malvindi PG, Bertani A, Romano G, Sciacca S, et al. Aortic surgery in Marfan patients with severe pectus excavatum. J Cardiovasc Med [Internet]. Mayo 2017; [citado 20 Agosto 2020]; 18(5):305-10. Disponible en: https:// pubmed.ncbi.nlm.nih.gov/27136701/

14. Baldassari E, Milanez J, Fernández A, Pinho L, Jatene F. Anthropometric index for Pectus excavatum. Clinics. 2007; 62(5): 599-606. DOl: http://dx.doi.org/10.1590/ S1807-59322007000500011.

15. García C, Mayorga A, Viteri F. Síndrome de Marfan y embarazo gemelar, presentación de un caso: Case report. Rev. Peru. ginecol. obstet [Internet]. Julio 2019 [citado 21 Diciembre 2020]; 65(3):345-8. Disponible en: $\quad$ https://doi.org/10.31403/rpgo. v66i2193

16. Martín $C$, Evangelista $A$, Serrano-Fiz $S$, Villar S, Ospina V, Martínez D, De Villarreal J, Sanchez V, Moñivas V, Mingo S, Forteza A. Aortic Complications in Marfan Syndrome, Should We Anticipate Preventive Aortic Root Surgery? Ann Thorac Surg [Internet]. Junio 2020; [citado 9 agosto 2020]; 109(6):1850-1857. Disponible en: https:// pubmed.ncbi.nlm.nih.gov/31589859/

17. von Kodolitsch Y, Demolder A, Girdauskas E, Kaemmerer $\mathrm{H}$, Kornhuber $\mathrm{K}$, Muino Mosquera $L$, et al. Features of Marfan syndrome not listed in the Ghent nosology - the dark side of the disease. Expert Rev Cardiovasc Ther [Internet]. Diciembre 2019; [citado 13 agosto 2020]; 17(12):883-915. Disponible en: https://pubmed.ncbi.nlm. nih.gov/31829751/
18. Gerovy E, Quiñones M, Acosta A. Síndrome de Marfán, a propósito de dos casos. Revista Finlay [Internet]. Febrero 2020; [citado 11 Agosto 2020]; 10(1):62-72. Disponible en: http://revfinlay.sld.cu/index. php/finlay/article/view/793 